\title{
Posterior reversible leukoencephalopathy syndrome (PRES) as a complication of ozone injections for chronic pain
}

\author{
Sarah H Sofka*, Troy M Krupica \\ West Virginia University School of Medicine, Morgantown, West Virginia, USA
}

Received: June 7, 2016

DOI: $10.5430 /$ crim.v3n3p65

\author{
Accepted: June 30, 2016 \\ Online Published: July 10, 2016 \\ URL: http://dx.doi.org/10.5430/crim.v3n3p65
}

\begin{abstract}
A 57-year-old female presented with encephalopathy, focal neurological findings, and possible seizure immediately after receiving an intramuscular injection of liquefied ozone to the posterior skull base. Ozone injections were being used to treat chronic neck pain and headaches. On presentation the patient was found to have a Glasgow Coma Scale score of 6. Her blood pressure was high at $220 / 93$ and she had a temperature of $39.5^{\circ} \mathrm{C}$. Neurological exam revealed increased muscle tone in the left leg as well as an extension plantar reflex. Lab work, including lumbar puncture results, were unremarkable. Magnetic resonance imaging (MRI) showed findings consistent with Posterior Reversible Leukoencephalopathy Syndrome (PRES). The patient's hypertension was treated and she significantly recovered after five days. Follow up MRI three weeks later showed interval resolution of the previous findings, and the patient had complete return to functional baseline thereby making the diagnosis of PRES most likely. No other cases of ozone induced PRES have been reported in the literature.
\end{abstract}

Key Words: Posterior reversible leukoencephalopathy syndrome, Ozone, Alternative medicine

\section{INTRODUCTION}

Ozone injections are a common type of modality used to treat back pain in Europe and Asia. Most commonly, ozone is used for pain related to disk herniation. Ozone is thought to have analgesic, anti-inflammatory, as well as oxidant action particularly in the nucleus pulposus. Observational studies and a few small randomized controlled trials, have shown pain reduction in patients treated with ozone injections. ${ }^{[1,2]}$ In the United States, ozone injections are not FDA approved for treatment of pain. However, many homeopathic and alternative medicine clinics are offering this treatment for musculoskeletal pain and headaches. There is little published about side effects or adverse outcomes of ozone therapy and generally it is thought to be very safe. ${ }^{[1,2]}$ This clinical report describes a 57-year-old woman who developed altered mental status, focal neurological deficits, and encephalopathy immediately after receiving ozone containing injections to the posterior skull base. We hypothesize that the ozone injections caused Posterior Reversible Leukoencephalopathy Syndrome (PRES).

\section{CASE REPORT}

A 57-year-old female with well controlled hypertension and chronic headaches, presented with altered mental status immediately after receiving an injection of liquefied ozone gas to the posterior skull base. The patient had been receiving

\footnotetext{
* Correspondence: Sarah H Sofka, Assistant Professor; Email: ssofka@ hsc.wvu.edu; Address: West Virginia University School of Medicine, Robert C. Byrd Health Science Center, Morgantown, WV 26506, USA.
} 
similar monthly injections for headaches and neck pain over the past 5 years. The injections contained lidocaine, procaine, dexamethasone, methylcobalamin and folic acid followed by an injection with liquefied ozone-oxygen gas at a concentration of $20 \mu \mathrm{g} / \mathrm{ml}$. After the ozone injection, the patient developed acute dizziness and left arm numbness. She then lost consciousness and was noted to have seizure like activity by witnesses. She was transported to the Emergency Department and regained consciousness but was disoriented and agitated.

Examination revealed a well-nourished female. She was febrile with a rectal temperature of $39.5^{\circ} \mathrm{C}$, elevated blood pressure of 220/93; heart rate of 101, respiratory rate of 16 and oxygen saturation of $97 \%$ on ambient air. Head was normocephalic and patient withdrew to pain with palpation of right posterior occiput. There was no evidence of superficial inflammation or abscess at the injection site. Neurologically, reflexes were $2+$ bilaterally in biceps, brachioradialis, patella, and Achilles tendons. Her Glasgow Coma Scale score was calculated at 6 with no eye opening $(+1)$, no verbal responses $(+1)$, and withdrawal from pain $(+4)$. Increased muscle tone was noted in the left lower extremity and plantar reflex was upgoing in the left lower extremity. The rest of the clinical findings were normal.

On initial investigation, complete blood count and basic metabolic panel were within normal limits including sodium, potassium, chloride, blood urea nitrogen, and creatinine. Total serum creatine kinase (CK) was 199 U/L (normal range 25-190 U/L). Toxicology screen was negative and blood alcohol level was zero. Urine analysis was negative. Urine drug screen was also negative. An unenhanced CT brain followed by contrast enhanced intracranial and extracranial angiographic images and perfusion images were obtained and revealed no abnormalities. A lumbar puncture was performed and revealed a clear, colorless fluid with an elevated protein at $80 \mathrm{mg} / \mathrm{dl}$ (range $15-45 \mathrm{mg} / \mathrm{dl}$ ), normal glucose, zero red blood cells and 1 nucleated cell. Opening pressure was normal at $12 \mathrm{~cm} / \mathrm{H}_{2} \mathrm{O}$. Cerebral spinal fluid gram stain was negative for organisms. Herpes simplex virus type 1 and 2 polymerase chain reaction (PCR) as well as varicella zoster PCR were also negative.

She underwent magnetic resonance imaging (MRI) of the brain with and without contrast that showed abnormal signal consistent with vasogenic edema in the gyri and subcortical white matter of the supratentorial brain including the occipital lobes. Findings were asymmetrically worse in the right frontal and right posterior temporal lobe (see Figure 1). Fluid Attenuated Inversion Recovery (FLAIR) and Apparent Diffusion Coefficient (ADP) mapping showed increased intensity in these areas. Differential for the findings included toxic demyelination and PRES.

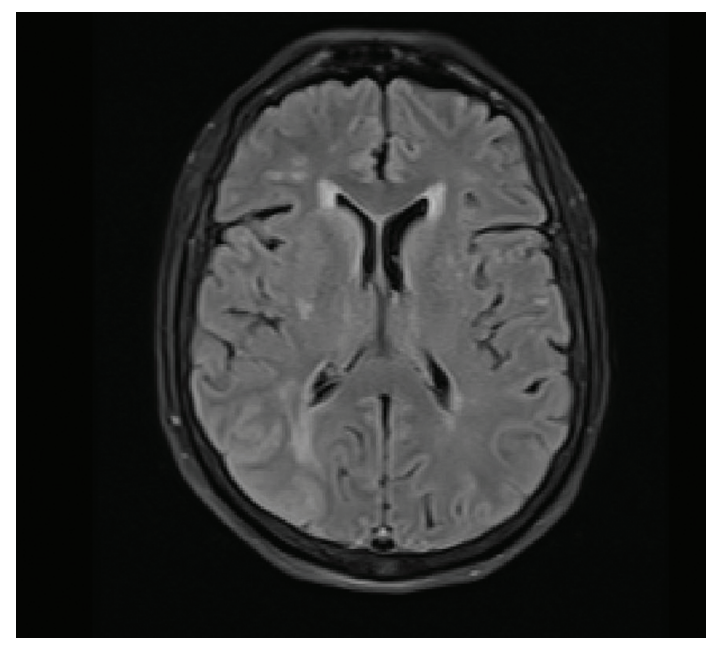

Figure 1. FLAIR MRI axial imaging at the time of presentation showing vasogenic edema in the gyri and subcortical white matter of the supratentorial brain, asymmetrically worse in the right frontal and right posterior temporal lobe. DWI and ADC mapping showed increased signal intensity in these areas

She was started on aggressive intravenous hydration of normal saline at $150 \mathrm{cc} / \mathrm{hr}$. She was placed on continuous electroencephalogram (EEG) with video monitoring. For her hypertension, she received one dose of $5 \mathrm{mg}$ intravenous labetalol and blood pressure normalized.

Her hospital course was essentially uncomplicated with the exception of persistent fever with temperatures ranging from 38.1 to 38.8 degrees Celsius. The patient's EEG revealed the presence of nonspecific diffuse slowing, being worse in the right hemisphere, as well as asymmetry with lower amplitude in the right hemisphere. No epileptiform activity was recorded. Over the next 36 hours, her CK gradually rose from $199 \mathrm{U} / \mathrm{L}$ to 413 to 573 to 752 . Her intravenous fluid was aggressively titrated to a maximum rate of $250 \mathrm{cc} / \mathrm{hr}$. Her creatinine and urine output remained normal. The CK then returned to normal.

The patient's physical examination remained unchanged for the first 24 hours; however, on day two the patient began spontaneously opening her eyes and followed simple commands such as hand squeeze. Mental status continued to rapidly improve over the next 12 hours and the patient began speaking and following all commands. She continued to be slightly confused and complained of a headache. At 48 hours, she was awake, alert, responding appropriately, and following all commands, however with slow interpretation and response times. She was evaluated by physical therapy 
and demonstrated decreased balance in sitting and standing and had some difficulty with steps. These deficits slowly resolved during her hospital admission. The patient was discharged on day five.

Follow up MRI three weeks later showed interval resolution of the previously seen abnormal cortical and subcortical signal in the cerebral hemispheres (see Figure 2). The resolution of the abnormalities was found to be consistent with a diagnosis of PRES.

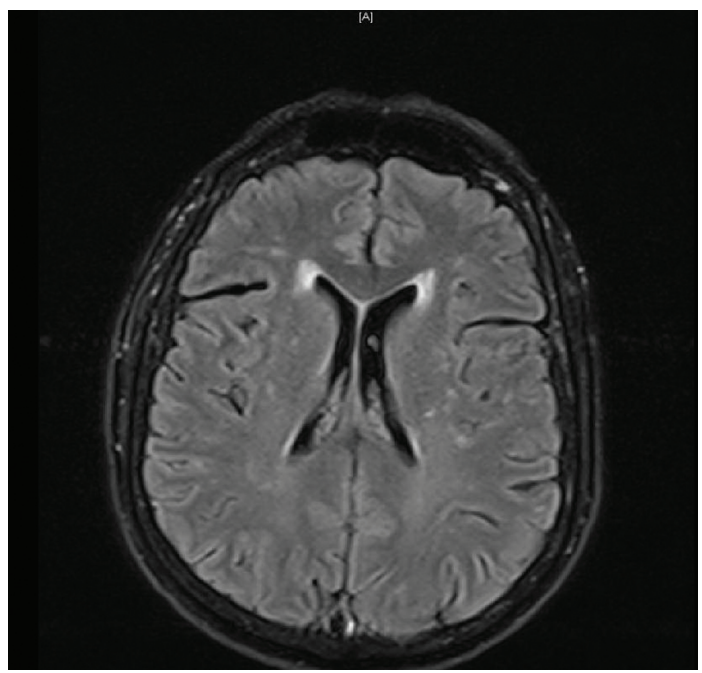

Figure 2. FLAIR MRI axial imaging three weeks after presentation showing interval resolution of the previously seen abnormal cortical and subcortical signal in the cerebral hemispheres

\section{Discussion}

This case is important to report as an increasing number of Americans seek out alternative medicine to treat chronic conditions. ${ }^{[3]}$ Ozone in particular is widely used in Europe and Asia for the treatment of pain related to disk herniation. A systematic review and meta-analysis demonstrated positive results and low morbidity rates in patient with low back pain related to herniated disk. ${ }^{[1]}$ In the United States, many alternative medicine practices are using ozone injections to treat other pain conditions including headaches, such as in our patient.

We supposed that a toxic dose of ozone may have been used or air may have been inadvertently injected into the CSF space. However, the concentration of ozone-oxygen mixture used in this patient was $20 \mu \mathrm{l} / \mathrm{ml}$ which is the standard dosing used in most published studies and is thought to be a therapeutic and non-toxic dose. ${ }^{[1,2]}$ Also, no evidence of pneumocephalus was seen on neuroimaging making this diagnosis unlikely.

We hypothesized that the ozone came in to contact with the blood brain barrier through inadvertent injection into the arterial circulation especially given the rapidity of symptom onset. Ozone is a powerful oxidizing agent and has been shown to damage the blood brain barrier in rats. ${ }^{[4]}$ The patient's clinical presentation including altered mental status, headache, and acutely elevated blood pressure are findings often seen with PRES. Seizure is also frequently reported in PRES. This patient likely had a seizure at presentation which explains her elevated CK levels. Neuroimaging is required for diagnosis and most commonly shows vasogenic, white matter edema predominately in the posterior lobes although many variations in the distribution of white matter edema have been reported including a large number of cases with frontal lobe involvement. Asymmetry has also been described in the literature and can be present in up to $50 \%$ of cases of PRES. ${ }^{[5]}$ Repeat imaging performed days to weeks later generally shows resolution of the changes. ${ }^{[5]}$ The etiology of PRES is thought to be a result of endothelial disruption and cerebral blood flow dysregulation which may be caused from a number of medical conditions as well as immunosuppressive and cytotoxic medications. ${ }^{[6]}$ We present this case as another possible cause of PRES and also to illustrate a dangerous side effect of cervical intramuscular ozone injections that has not previously been reported in the literature.

\section{Conclusion}

Ozone injections, which are gaining popularity in the world of alternative medicine for treating chronic pain, may cause unforeseen complications. In this clinical case, a patient receiving ozone injections to the base of the skull, developed PRES as complication of this alternative therapy.

\section{CONFlicts OF InTEREST Disclosure}

The authors have declared no conflicts of interest.

\section{REFERENCES}

[1] De Oliveira Magalhaes F, Dotta L, Sasse A, et al. Ozone Therapy as a Treatment for Low Back Pain Secondary to Herniated Disc: A Systematic Review and Meta-analysis of Randomized Controlled
Trials. Pain Physician. 2012; 15: E115-E129.

[2] Paoloni M, Di Sante L, Cachio A, et al. Intramuscular OxygenOzone Therapy in the Treatment of Acute Back Pain with Lumbar Disk Herniation. Spine. 2009; 34: 1337-1344. PMid:19478653 
http://dx.doi.org/10.1097/BRS.0b013e3181a3c18d

[3] Weirtheimer A, Brentson F. Homeopathic Medicine Should Have a Role in Managed Care. Managed Care. 2014; 23(11): 38-42.

[4] Rivas-Arancibia S, Hernández-Zimbrón L, Rodríguez-Martínez E. Chronic Exposure to Low Dose Ozone Produces a State of Oxidative Stress and Blood-brain Barrier Damage in the Hippocampus of Rats. Advances in Bioscience and Biotechnology. 2013; 4: 24-29. http://dx.doi.org/10.4236/abb. 2013.411A2004
[5] Fugate J, Claassen D, Cloft H, et al. Posterior Reversible Encephalopathy Syndrome: Associated Clinical and Radiologic Findings. Mayo Clinic Proceedings. 2010; 85(5): $427-$ 432. PMid:20435835 http://dx.doi.org/10.4065/mcp. 2009. 0590

[6] Hinchey J, Chaves C, Appignani B, et al. A Reversible Posterior Leukoencephalopathy Syndrome. New England Journal of Medicine. 1996; 334(8): 494-500. PMid:8559202 http://dx.doi.org/10. 1056/NEJM199602223340803 